\title{
Security procedures and devices for road transportation of high consequence dangerous goods
}

\author{
A. Accettura ${ }^{1}$, R. Bubbico ${ }^{2}$, F. Garzia ${ }^{3,4}$ \& B. Mazzarotta ${ }^{2}$ \\ ${ }^{I}$ Dipartimento di Ingegneria Civile, Edile e Ambientale, \\ Università di Roma "La Sapienza", Italy \\ ${ }^{2}$ Dipartimento di Ingegneria Chimica, Materiali, Ambiente, \\ Università di Roma "La Sapienza", Italy \\ ${ }^{3}$ Dipartimento di Ingegneria dell'Informazione, Elettronica e \\ Telecomunicazioni, Università di Roma "La Sapienza", Italy \\ ${ }^{4}$ Wessex Institute of Technology, Southampton, UK
}

\begin{abstract}
Road tankers transporting high consequences dangerous goods (HCDG) may represent an attractive target for terrorists, travelling practically anywhere, without escort, and presenting the potential to give rise to catastrophic incidents with a number of fatalities. Different kinds of security devices are available, ensuring a number of useful functions (localization of the vehicle, communication, etc.): their cost may represent a problem, even if prices are progressively decreasing, but additional concern may be workers' privacy, which should be safeguarded. The operating procedures should be carefully tuned to provide the needed protection level without bothering the driver with a number of time consuming tasks to be fulfilled. The driver may be taken as hostage by terrorists, and forced to obey to their orders, this eventuality should be assessed without endangering the worker's life. The present work outlines some basic arrangements of security devices and proposes some procedures to prevent threats to security posed by road transportation of HCDG.

Keywords: security, road transport, hazardous materials, high consequences dangerous goods, security procedure, security devices, terrorist attack.
\end{abstract}




\section{Introduction}

The transportation of hazardous materials (HazMat) has been addressed by many authors in the recent years from different point of views: accident prevention, safety for the exposed population, optimization of the route, and so on. Furthermore, in the very recent years (especially after the event of September 11, 2001), terrorist activity possibly related with the transportation of sensible and hazardous materials, has been recognized as a significant threat to public safety, and increasing attention has been devoted to the specific issue of the security in HazMat transportation (Abkowitz [1]; TRB [2]). In fact, on the one hand, it is generally assumed that a high level of technical expertise and knowledge, as well as highly sophisticated equipment and tools, are not needed for such a kind of action, despite the catastrophic consequences that might be generated after the release of the material (sometimes considered at the same level of weapons of mass destruction). On the other hand, given the complexity of the logistics and the non-standardized shipment procedure, a large number of sensible exposure points are present, and since not all of those critical points can be totally eliminated, the overall vulnerability of this kind of activity cannot be reduced to zero. As a consequence, even though the use of traditional explosives is still the preferred technique in terrorist activities and the main concern for the Authorities is usually based on surmises and generic information, the above considerations make the use of more conventional materials quite attractive. This is also witnessed by recent events, such as the London and Glasgow attacks (both fortunately unsuccessful) in 2007, as well as by many other events, outside the terrorism area, but still within the criminal activity, as reported by newspapers almost daily.

Different approaches have been adopted in the literature to reduce the risk associated with HazMat transportation: risk analysis, vulnerability analysis, cost/benefits analysis and so on. In particular, the issue of security in the transportation of HazMat has been addressed either in the framework of a more general approach (an "extended" risk analysis, see for example Huang and Cheu [3] and Huang et al. [4]) or considered alone. It has been properly observed that the inclusion of security in a more general approach is not straightforward, due to a number of peculiarities: the probability of release (in this case the attack probability) is not uniform (Erkut et al. [5]), but is dependent, also but not exclusively, on what we might call the "attractiveness" of the location, characterized by the expected damages.

However, the problem of accounting for security aspects of the transportation of hazardous materials is even more complex. The term "hazardous material" can cover a broad range of products with very different characteristics: it is usually adopted for flammable substances (liquids or gases), explosives, and toxic materials. However, depending on the target, on the availability and on the capabilities of the terrorists it can also include generic poisonous materials, such as pesticides (for example, to contaminate water sources), oxidizing or corrosive substances, or even, though more rarely, nuclear or biological materials. The use of one or the other among those materials can depend either on its availability or 
easiness of coming into possession, or on the intended consequences. The main consequence caused by flammable materials is a fire. In the case of liquids, the fire is intense, rather localized, and long-lasting, so that it can cause serious structural damages (both to metal and concrete structures), ignite further substances and impact people if not able to escape. In the case of a gas, similar localized consequences are obtained following the immediate ignition of a substantial catastrophic release of a flammable mass (fireball), even though, here, the influence of buoyancy is important; conversely, in the case of a delayed ignition, the fire is markedly influenced by the atmospheric conditions (wind velocity and direction, presence of obstacles, and so on), so that even locations relatively far away from the release site can be impacted. Furthermore, even though only under proper conditions, in the case of a gas cloud a much stronger combustion with explosive effects (VCE, Vapour Cloud Explosion) can occur. In this case, the consequences will be more serious, and will usually cover larger distances, compared with the case of a common fire. On the other hand, since a number of external conditions need to be met at the same time, its probability of occurrence is lower. However, the chemical reactivity of the specific substance plays here a fundamental role and, for highly reactive materials, the above external conditions can be less restrictive.

Differently from the material types mentioned so far, which usually cause damages both to structures and people, toxic substances represent a rather different case since, when released into the atmosphere, they have almost no effect on the structures and environment, but, due to the very large impact areas possibly generated, they can cause very high numbers of casualties and injuries within the population. Under this respect, gases and low-boiling (high volatility) liquids are most dangerous, however, besides their physical conditions, the most important parameter is represented by the toxicity of the particular substance. Finally, for a given cloud extension, the area actually impacted by the released chemical is strongly influenced by the weather and wind conditions, being thus quite unpredictable without detailed on-time data. However, when a toxic chemical is released in the open, its concentration inside buildings (so-called indoor concentration), assuming that doors and windows are closed, will hardly reach very high and dangerous values, being much lower than outside; on the other hand, while in the open the substance will be rapidly diluted with air, in closed spaces with limited air recirculation rates, the lower concentration will persist for longer thus increasing the exposure time. As an alternative scenario, if a toxic substance is released directly within a confined space (for example, a tunnel, a building, etc.) it will cause the worst effects on the exposed population, due to the combination of high concentration (caused by a lower dilution with air) and longer exposure time.

Of course the choice of a specific hazardous material by candidate terrorists, will depend on many different parameters: the availability of the chemical, the skill, knowledge and level of organization of the terrorists, the security level of the transportation company, possible insider collusion, kind of result sought, and so on. 
With specific reference to the security issue, a number of projects, based on very practical approaches, have been implemented in the recent years.

The U.S. Research and Special Programs Administration (RSPA), a division of the U.S. Department of Transportation (DOT), has asked all those involved in this activity (during transport, storage, etc.) to review and, if necessary, improve their security measures (US DOT [6]). The recommendations and security requirement issued by the US RSPA, cover many aspects of the transportation activity: registration certificates, personnel security and training, facility security, security plans, documentation, en-route security and others.

In a recent study (De Lorenzo et al. [7]), the influence of different available technologies on various aspects of the transportation of hazardous materials have been analysed, ranging from safety, to security and efficiency. A strong positive effect was found after the introduction of wireless communication systems and GPS positioning, not only in terms of vulnerability reduction, but also of a higher benefit/cost return. Furthermore, the reduction in the information exchange time, also has a marked benefit on the emergency and security response.

With specific reference to the vulnerability issue, which is considered as one of the three main aspects related with security, together with threat and consequences, it was found that increasing benefits can be obtained by adopting an increasing number of technological tools, i.e. wireless communication, panic buttons, and vehicle disabling, respectively.

In 2007-2008, the Kentucky Transportation Center of the University of Kentucky, carried out a project aimed at assessing the feasibility of establishing a Transportation Security Center (TSC), to be operating in North America (University of Kentucky [8]). The transportation fleet should be composed of "smart trucks" equipped with on-board computers, GPS receiver and wireless connection. The internet connection with both the Transportation Security Center and the fleet tracking data center will allow a continuous knowledge of the location of the truck at any time, as well as of the type and quantities of the material transported. The pre-selected route plan can be electronically submitted to the TSC and alarms can be generated in case of unexpected events.

The introduction of further security systems, based on well-known technologies already adopted in higher-value materials transportation (e.g. cigarettes!), such as vehicle tracking and remote immobilization, has been strongly supported in a recent and comprehensive report by the Mineta Transportation Institute (MTI [9]) where a critical and detailed analysis of both the possible aims of a terrorist organization and the available means (also in terms of seizing easiness) is reported.

\section{Security aspects of road transport of hazardous materials}

In Europe, road transport of hazardous substances is ruled by the ADR code (ECE [10]), which divides these products into 9 main classes, and includes provisions concerning packing and tanks, consignment, requirements for construction and testing, condition of carriage, loading, unloading and handling, requirements for vehicle crews, equipment, operation and documentation, or 
concerning the construction and approval of vehicles, special provisions and exemptions related to dangerous goods packed in limited quantities. ADR also includes some specific security provisions (Chapter 1.10), intended as measures or precautions to be taken to minimize theft or misuse of dangerous goods that may endanger persons, property or the environment. It is required to properly identify and register carriers, securing temporary storage sites and performing safety inspections, and to provide involved personnel with specific security awareness training about security risks, their nature, methods to recognize, address and reduce such risks and actions to be taken in the event of a security breach. A list of "high consequences" dangerous goods (HCDG) having the potential for misuse in a terrorist incident and which may, as a result, produce serious consequences such as mass casualties or mass destruction is issued. HCDGs include all classes from 1 to 8 , some of which only if belonging to packaging groups I and II, for any quantity in case of highly hazardous products (such as toxics), or in quantities exceeding 3000 liters for less hazardous products (such as flammable liquids and gases, and corrosive substances). Carriers and other people engaged in the carriage of HCDG shall adopt, implement and comply with a security plan addressing some specific elements: a) specific allocation of responsibilities for security to qualified persons with appropriate authority to carry out their responsibilities; b) records of dangerous goods concerned; c) review of current operations and assessment of security risks (including stops, keeping of dangerous goods in the vehicle, intermediate temporary storage of dangerous goods); d) clear statement of measures that are to be taken to reduce security risks, commensurate with the responsibilities and duties of the participant (training, security policies, operating practices, equipment and resources to reduce security risks); e) effective and up to date procedures for reporting and dealing with security threats, breaches of security or security incidents; f) procedures for evaluating security plans and procedures for periodic review; g) measures to ensure the physical security of transport information contained in the security plan; h) measures to ensure that the distribution of information relating to the transport operation contained in the security plan is limited to those who actually need to have it.

It is required to apply all measures to prevent the theft of a vehicle carrying HCDG, to control that the devices are properly operating and, when appropriate and already fitted, to use tracking apparatus to monitor the movement of HCDGs.

In the USA, the Department of Transport issued an Hazardous Materials Regulation (HMR) requiring persons who transport a given amount of certain hazardous materials (for example, $55 \mathrm{~kg}$ of explosives, $1 \mathrm{~L}$ of material poisonous per inhalation, $13.248 \mathrm{~m}^{3}$ of gas, liquids and solids) to develop and implement security plans (DOT [11]): these materials include quantities exceeding 3000 liters for less hazardous product (such as flammable liquids and gases, and corrosive substances). In order to making a rule (DOT [12]) a qualitative risk evaluation was carried out, which considered the following factors: (1) physical and chemical properties of the material and how those properties could contribute to a security incident; (2) quantities shipped and mode of transport; 
(3) past terrorist use; (4) potential use; (5) availability. One of the most significant security vulnerabilities involves the potential for an individual or group to take control of a conveyance containing a high-risk material and move it to a site where the material could cause maximum physical or psychological damage. For some hazardous materials, the primary security threat involves theft or hijacking of raw materials for use in developing explosive devices or weapons. The final list of hazardous substances requiring a security plan is almost the same that defined by the ADR.

It is obvious from HazMat transportation data that vehicles carrying HCDGs may easily reach percentages well exceeding $70 \%$ of the total, mainly due to the presence of liquid and liquefied fuels in the list. Therefore, it may be appropriate to further group HCDGs based on their expected attractiveness for terrorists, usually defined in terms of their potential for producing catastrophic consequences and large impact areas. Chemicals susceptible to explode (such as substances belonging to ADR Class 1 or Class 5.1, but not Classes 4.1, 4.2 and 4.3) are, obviously, the best candidates, while corrosive or toxic substances (such as ADR Class 8, 6.1 or 6.2) appear less attractive, based on the considerations summarized in section 1. As far as the remaining classes are concerned, flammable gases (belonging to Class 2) are susceptible to explode throwing fragments of the vessel at some hundred meters, thus resulting more suitable for terrorist uses than flammable liquids (Class 3). The transport of radioactive materials (for civil uses) is not considered here, since it is already subject to a number of limitations and precautions and may be assumed as safe enough.

In examining security aspects of hazardous materials transportation, it should be considered that, presently, routes may cross built-up areas, as well as brush past crowded locations. This occurs especially when gaseous and/or liquids fuels are distributed to scattered users within towns, and makes rather complicated to avoid the tankers passing close to any populated site. As a matter of fact, in a number of cases a road accident occurred to a truck transporting hazardous substances has originated multiple fatalities following the (non intentional) release of the product: for example, in the well-known accident of San Carlos de la Rapita (Spain) of 1978, the ignition of propylene spilled from an overfilled tanker killed more than 200 persons in a camping site.

Concerning, in particular, terrorism groups and the existent threat they would use HazMat transports, it is not so much easy to say if this will really tempt such organizations; however, it is well-known that terrorists "passions" are the means of transports in all their facets. Terrorism is a dynamic human phenomenon (Ganor, 2005), ready to change its shapes according to geographic position and time of acting. In order to carry out a risk and vulnerability analysis for HazMat transports a number of parameters should be listed and analyzed (Barilla, 2009): 1) the legal or illegal possibility to collect explosives, or chemicals which can be mixed to give an explosive, in order to make homemade bombs; 2) the ability of making operable and efficient IEDs (Improvised Explosive Devices), which depends on the availability of appropriate know-how and technology; 3) the easiness of attacking a HazMat transporting vehicle, which may depend on the frequency of passage and on the presence of measures of prevention; 4) the 
possibility for a HazMat transporting vehicle to become a weapon, and the fatalities it can cause, which depend, primarily, on the characteristics of the chemicals, but also on the location where its content might be intentionally released. A recent paper (Milazzo et al. [13]) focuses attention on the emergency management following a terrorist attack in the transport of hazardous materials in urban areas.

\section{Security procedures and devices}

In this section, a series of measures and procedures for improving the security in the transportation of hazardous materials will be proposed. It is likely that some of the proposed measures are not immediately applicable to every type of transportation means (especially for relatively low-value and widely transported materials, like gasoline and propane, of for smaller transportation companies), but at least for more sensible/hazardous materials, they should be definitely taken into consideration. In this view, a preliminary classification of the many HazMats characterized by different levels of importance would be very useful. The list of HCDGs, in fact, includes too many products (e.g. fuels) and transport activities, so that it is not thinkable, at the moment, to provide each vehicle with all the suggested safety devices or to implement all the proposed security procedures. From this point of view, a preliminary guideline in order to properly scale the amount of devices and the complexity of procedures to be adopted in each specific case, may be grouping the HCDGs according to the rank suggested in the previous section.

The importance of the choice of most proper technological devices to be applied to HazMat transport has been already treated by Abkowitz and Tate [14]: the authors developed a methodology to screen and select the best available technologies to enhance HazMat transportation safety and security, according to the different areas of application.

The first type of safety devices to be considered (as suggested by ADR) is a tracking apparatus, such as a GPS (Global Positioning System) to monitor the movement of the HCDG. Presently, in Italy, GPSs are not routinely installed on all vehicles transporting HazMats. This choice should be motivated based on financial resources and flexibility at work, but such devices were already considered suitable in the past for particular scenarios (for example theaters of war, such as Afghanistan). On the civilian side, the use of GPS systems may be useful, especially when the vehicle has to deliver goods to different spots, but problems arise concerning the workers' privacy. Security can clash with privacy, and their relationships should be properly taken into account, since cargo tracking is the first aspect to be considered in HazMat transport security.

However, GPSs are not the only devices which can and should be used: mobile phones, touch screen displays, cameras, microphones, fingerprint detection and so on, can also be considered, among others.

Another important aspect to be considered is the possible inattention caused by the use of hi-tech devices while driving: US DOT (Shutko et al. [15]) investigated the impact of cell phones use on drivers' attention (focusing on text 
messaging in particular), on driving performances, and on possible consequent road accidents.

With all these premises in mind, the proposed devices and procedures for improving the security when transporting HCDG products can be represented by a "security box".

The proposed system is composed of a central module and a series of additional functional modules, which can be added or not at any time, according to the requested protection level for the vehicle under consideration. The central module can also integrate already existing functional modules to reduce the final cost as much as possible. It will be powered by an internal battery, recharged by the electrical supply of the vehicle, in order to be totally autonomous and to be working even in the case of loss of the main electrical supply.

The functional modules are the following:

1. GPS: a normal positioning receiver, providing the geographic coordinates both to the driver and to the remote operator.

2. Cellular communication: used to transmit to the remote operator the geographic coordinates and the data (alarm, videos, etc.).

3. Satellite communication: operating as a redundant channel with respect to the cellular communication module, intended as primary transmission channel in rural areas, where the cellular service could not be guaranteed.

4. Human interface, composed by touch screen, biometric interface (fingerprint detection) and emergency buttons; it is intended to be used mainly by the drivers.

5. Vehicle velocity: it allows the remote operator to know the velocity of the vehicle.

6. Front-rear vehicle distance: it allows the remote operator to identify when the vehicle is trapped in a traffic-jam.

7. Bumping: it allows the remote operator to autonomously give an alert in case of incident.

8. Theft detection: this module can be used either by the driver or by the remote operator to check and prevent theft of the vehicle and of the transported material.

9. Video surveillance: composed by an internal camera, providing images of what is happening in the cockpit, and by four external cameras, located on the front side, on the rear side and on the two lateral sides. The images are transmitted, in emergency situation by means of the available communication channels (cellular, satellite), to the remote operator.

Thanks to its modular and flexible structure, it is possible to scale the complete security module into three security boxes, characterized by different levels of functionalities, according to the hazard posed by the materials transported by the vehicle to protect. The characteristics of these different security boxes are shown in Table 1, together with the related functionalities and the indicative cost.

The security boxes have been thought of and designed to face different kinds of attacks, trying to imagine the different situations which might happen during the journey of the vehicle and the places where these scenarios might be put into 
action, with the objective to prevent those events from occurring and, at the same time, not to complicate the driver's work too much, and to preserve his/her privacy.

Table 1: $\quad$ Characteristics of the security boxes.

\begin{tabular}{|l|l|c|}
\hline Level & Functionalities & Approximate cost \\
\hline Basic & - GPS module & $€ 1000$ \\
& - cellular communication module & \\
& - human interface module & $€ 2200$ \\
\hline Intermediate & The same as Basic level plus: & \\
& - vehicle velocity module; & \\
& - front-rear vehicle distance module; & \\
& - bumping module; & $€ 3500$ \\
\hline Advanced & $\begin{array}{l}\text { The same as Intermediate level plus: } \\
\text { satellite communication module; } \\
\end{array}$ & \\
& video surveillance module. & \\
\hline
\end{tabular}

The core of the system is a remote central control room, which is supposed to be looked after and monitored by a $24 / 7$ real time operator (of course a shorter period might be adopted depending on the needs of the specific transportation case). The room operator should register and watch over all the data concerning the HCDG vehicle trip, before and during the motion, receive the alarms, activate all the remote operating devices, and interact with the driver, when needed or requested. The tasks are not expected to be very demanding; however a specific training for the operator will be required.

With reference to the driver, the first task he is required to do, before getting into the vehicle, is to accurately plan the route and to inform the remote control operator/system about this route through the GPS the tanker is equipped with. The path to reach the destination should be chosen in a very coherent and safe way, avoiding (as much as possible) built-up areas, schools, critical points and any other places which could put in danger many people, should a spill occur. If the path is changed without informing the remote control an alarm will start automatically. The second step the driver must comply with is the fingerprint checking, which is the only way to check that the right -driver is on the rightvehicle. After passing this check the vehicle can be set in motion: however, within the following 2 minutes, the driver should also push a hidden safety start button. This is intended as a measure to guarantee the driver safety, if under threat by terrorists or criminals: if the button is not pressed after the biometric control confirmation, an alarm starts and the vehicle is automatically decelerated as long as it stops.

After the biometric check and the activation of the start button the vehicle is in regime motion, and a number of different controls will follow its travel.

Should an accident occur to the vehicle, the bumping detection module will automatically give an alarm. Moreover, the driver should immediately inform the 
remote control operator about route change requirement (for example due to ordinary traffic causes), otherwise an alarm will start.

Tunnels are potentially critical spots, since GPS signal is not available there: however, the GPS, which knows the entire path, including the tunnel's length, can estimate the time required to travel through the tunnel. Assuming $30 \mathrm{~km} / \mathrm{h}$ as a suitable speed for the vehicle in the tunnel, if the vehicle takes longer than the expected time, the central operator will call the driver to check about the situation (this procedure will be applied only to tunnels longer than $1 \mathrm{~km}$ ).

Another issue to be considered is the need of stopping the vehicle during the trip, to allow the driver to eat, take a rest, or any other reason. In this case, the remote control operator/system, which can monitor the real time vehicles situation, will know that the vehicle is not in motion: if after a reasonable break time (for example, $45 \mathrm{~min}$ ) the vehicles is still parked, an alarm is given to the remote control operator, who will call the driver to know what is happening.

Basically, in this procedure, different scenarios can happen: the alarm might start automatically, or a contact might be required between driver and remote control operator, or in other cases the alarm responsibility will be on the driver. Every time an alarm is given, it is assumed that an emergency situation is in progress, and the remote control operator will switch audio (microphones) and video (inside and outside cameras) modules on: then the remote control operator can have a clear idea of what is occurring, in that moment, inside the cab.

It is clear enough that procedure laboriousness and complexity can bother the driver somehow, (not used to and reluctant to apply changes) but, at the same time, the application of prevention measures should not find hostilities.

\section{Conclusion}

In the present paper some critical issues related with the security during transportation of dangerous materials have been addressed. Most of those materials are widely used in everyday life, but, in case of release, severe consequences to the exposed population can be expected, depending on the type of material and on the accidental conditions. This makes them very attractive from a terrorist's point of view. Even if the risk associated with possible terrorist attacks or other criminal actions cannot be reduced to zero, nonetheless a proper security analysis can help identifying critical spots in terms of vulnerability and consequently allow the adoption of adequate prevention measures. It is believed that the proposed procedures, which are based on already easily available technologies, can markedly improve the security of many transportation activities, in particular thanks to its flexible and modular structure. This characteristic, in fact, makes it possible to adapt the complexity of the procedure, and of the supporting technologies, to the particular needs and to the level of hazard posed by the specific transportation case under investigation. Besides the main issue of the vulnerability to external threats, other aspects have also been taken into consideration, aiming at limiting the complexity of the procedure and its interference with the ordinary activities during transfer and to protect the privacy of the involved working personnel. Since a widespread and full 
application of the proposed procedure will be probably limited by budget reasons only, a preliminary cost ranking (at the date of writing) has been presented, but it is likely that thanks to the continuously decreasing costs of the hardware components of the security system, even smaller companies, local or short range transfers, or less hazardous materials, will benefit from its adoption, with a marked reduction of the target vulnerability and a corresponding increase of the security for the possibly exposed population.

\section{References}

[1] Abkowitz, M.D., Transportation risk management: A new paradigm. Security papers, Southeastern Transportation Center, University of Tennessee, pp. 93-103, 2002

[2] TRB, Deterrence, protection, and preparation: The new transportation security imperative. Special Report 270, Transportation Research Board, 2002

[3] Huang, B., Cheu, R.L., GIS and genetic algorithms for HAZMAT route planning with security considerations. International Journal of Geographical Information Science 18 (8), 769-787, 2004

[4] Huang, B., Long, C.R., Liew, Y.S., GIS - AHP model for HAZMAT routing with security considerations. In: IEEE 6th Int'l Conf. on ITS (ITSC2003), 2003

[5] Erkut, E., Tjandra, S.A., Verter, V., Hazardous materials transportation, in Handbook in OR\&MS, vol.14, Ch. 9, C. Barnhart and G. Laporte Eds., Elsevier BV, 2007

[6] US DOT, Department of Transportation, Research and Special Programs Administration. Advisory notice: Enhancing the security of hazardous materials in transportation. 67 Federal Register 6963-6966, 2002

[7] De Lorenzo, J.P., Allen, J., Williams, D., Jensen, M., Safety, security and efficiency benefits of technology in highway hazardous materials transportation applications, Proceedings of the 2005 Mid-continent transportation research symposium, Ames, Iowa, August 2005

[8] University of Kentucky, Kentucky Transportation Center, UKRF 3048103539-08-017, December 2008

[9] MTI, Mineta Transportation Institute, Potential Terrorist Uses of HighwayBorne Hazardous Materials, Report n. 09-03, 2010

[10] ECE, European Commission for Europe - Committee on Inland Transportation, ADR, International Agreement Concerning the International Carriage of Dangerous Goods by Road, ECE/TRANS/215, United Nations, Geneva

[11] US DOT, Department of Transportation, Interim Final Rule [IFR], Enhancing rail transportation safety and security for hazardous materials. 73 FR 72181 - Final Rule, 2008

[12] US DOT, Department Of Transport - Pipeline and Hazardous Materials Safety Administration, 49 CFR Part 172. Hazardous Materials: Risk-Based 
Adjustment of Transportation Security Plan Requirements. Federal Register / Vol. 75, No. 45 / Tuesday, March 9, 2010 / Rules and Regulations, 2010

[13] Milazzo, M.F., Ancione, G., Lisi, R., Vianello, C., Maschio, G., Risk management of terrorist attacks in the transport of hazardous materials using dynamic geoevents. Journal of Loss Prevention in the Process Industries 22, 625-633, 2009

[14] Abkowitz, M.D., W.H., Tate, Hazardous Materials Cooperative Research Program Report 4, 2011

[15] Shutko, J., Mayer, K., Laansoo, E., and Tijerina, L., Driver Workload Effects of Cell Phone, Music Player, and Text Messaging Tasks with the Ford SYNC Voice Interface versus Handheld Visual-Manual Interfaces, SAE Technical Paper 2009-01-0786, 2009

[16] Barilla, D., Leonardi, G., Puglisi, A., Risk Assessment for Hazardous Materials Transportation, in Applied Mathematical Sciences, Vol. 3, no. 46, pp. $2295-2309,2009$

[17] Ganor, B., The Counter-Terrorism Puzzle: A guide for Decision Makers, New Brunswick, N.J.: Transactions, 2005

[18] Glaze, M., New security requirements for hazmat transportation. Occupational Health \& Safety 72 (9), 182-185, 2003

[19] Federchimica, $5^{\circ}$ Rapporto S.E.T. Servizio Emergenze Trasporti - Anno 2011. Centro Stampa Accademia, Milano, pp. 2-7, 2012

[20] Jenkins, B.M., Notes from classes Summer Course of Counter Terrorism Studies, Interdisciplinary Center of Herzliya, Israel, 2011

[21] Rubin, H., Shahar, Y., Ganor, B., Wagner, A., Hellenthal, M., Popovich, E., What's next? Future Terrorist Scenarios and Technological Means to Counter Them, workshop, 11th ICT's World Summit on Counter Terrorism, Herzliya (Israel), 2011

[22] Tate, W.H., Abkowitz, M.D. Emerging technologies applicable to hazardous materials transportation safety and security, HMCRP Report 4 (Hazardous Materials Cooperative Research Program), 2011

[23] U.S. DOT, Department Of Transport, Code of Federal Legislation 49 Transportation, U.S. Government Printing Office, Washington, 2006

[24] Zografos, K.G., Androutsopoulos, K.N., Assessing impacts from introduction of advanced transport telematics technologies in hazardous materials fleet management. In: Proceedings of the 80th Annual Meeting of Transportation Research Board, Washington DC, USA, 2001 